parallel to each other in the central arm of an ' $\mathrm{H}$ '. This study aimed to determine the population variance in chiasmal shape.

Methods 68 MRI scans of healthy individuals without visual abnormality were randomly selected. A 2D image was created and images were analysed using AutoCAD software to determine the offset between lines drawn down the centres of the optic nerves and contralateral optic tracts. A positive offset would suggest an ' $H$ ' shape while an ' $\mathrm{X}$ '-shaped chiasm would have a offset of 0 .

Results The mean width of the chiasm was $12.0 \mathrm{~mm}$, and the mean offset was $4.7 \mathrm{~mm}$ generating a mean offset:width ratio of 0.38 . No chiasm had an offset of zero. Fibre crossings occurred approximately $2.35 \mathrm{~mm}$ lateral to the midline, and nasal (crossing) fibres travelled an average of $4.7 \mathrm{~mm}$ in the mediolateral plane before entering the contralateral optic tract.

Conclusions The human optic chiasm is H-shaped, not $\mathrm{X}$ shaped. This information will inform future models of chiasmal compression.

\section{WILL REFRACTORY PATIENTS RESPOND TO ERENUMAB IN THE REAL WORLD?}

${ }^{1}$ Bronwyn Jenkins*, ${ }^{2}$ Shuli Cheng, ${ }^{3}$ Elspeth Hutton. ${ }^{1}$ Neurology, Royal North Shore Hospital, St Leonards, NSW, Australia; ${ }^{2}$ Neurology, Alfred Hospital, Melbourne, VIC, Australia; ${ }^{3}$ Neurology, Alfred Hospital, Monash University, Melbourne, VIC, Australia

10.1136/jnnp-2019-anzan.26

Introduction Erenumab has been studied in Randomised Controlled Trials (RCT), with stricter exclusion criteria than real world populations.

Methods 65 patients from two Australian headache centres on Erenumab had primary outcomes of $\geq 50 \%$ response in monthly migraine days (MMD) and monthly headache days (MHD), compared to their demographics, frequency, duration of Chronic Migraine (CM), failed prophylactic medications, severity scores and medication overuse headache $(\mathrm{MOH})$.

Results 35\% (23/65) had daily headaches, with $\geq 50 \%$ MHD and MMD reduction in this subgroup of $17 \%$ (4/23) and $65 \%(13 / 20)$, respectively. Duration of CM was $>10$ years in $48 \%$ (29/61), with $\geq 50 \%$ MHD and MMD reduction in $28 \%(8 / 29)$ and $48 \%(13 / 27)$, respectively. $100 \%(64 / 64)$ had failed $>3$ prophylactic medications with $\geq 50 \%$ MHD and MMD reduction in $30 \%(19 / 64)$ and $47 \%$ (27/58), respectively, with reducing rates of $\geq 50 \% \mathrm{MMD}$ reduction if $>5$ $(29 \% ; 17 / 58)$ and $>10(22 \% ; 2 / 9)$. 95\% had high severity scores (HIT-6 score $>60$ ), with a $\geq 50 \%$ MMD reduction in $43 \%$ (23/54). $\mathrm{MOH}$ occurred in $41 \%$ (24/58) for triptan and $29 \%(17 / 58)$ for codeine medications, with $a \geq 50 \%$ MMD reduction in $71 \%(17 / 24)$ and $41 \%$ (7/17), respectively (all groups $\mathrm{p}>0.05)$.

Conclusion This real world cohort treated with Erenumab included patients that would be excluded from RCT analysis- including more chronic, frequent, severe and refractory migraine. Despite this, there were still $\geq 50 \%$ responders in more severe subgroups, particularly daily headache, high severity scores and triptan MOH. Measuring MMD may be more sensitive for assessing improvement than MHD.

\section{Poster abstract}

\section{DELAYED CT IMAGING LEADING TO DELAYS IN ACUTE STROKE MANAGEMENT IN REGIONAL AUSTRALIA}

Udit Nindra*, Toni M Wonson, Karen Fuller. Neurology Department, Wollongong Hospital, Wollongong, NSW, Australia

\subsection{6/jnnp-2019-anzan.27}

Introduction Urgent CT imaging is crucial for acute stroke management to allow for timely thrombolysis and early referral to a peripheral endovascular thrombectomy (ECR) service. Delays in CT imaging are suspected to correlate with lengthening door-to-needle time (DNT) and arrival-to-referral time (ART) in regional Australia.

Methods and results We retrospectively analysed 656 acute stroke admissions between 2016 and 2018 to determine mean DNT and ART in addition to influencing factors such as age, gender, onset to arrival time \& baseline National Institute of Health Stroke Scale (NIHSS) score. Over 3 years, 70 patients underwent thrombolysis and 56 ECR. The mean DNT was 108 minutes with mean arrival to CT time of 30 minutes. Multiple linear regression displayed a positive correlation between arrival to CT time and DNT $(p<0.01)$. For every 10 -minute delay in CT imaging, there was a 6-minute delay in DNT (95\% CI 2 - 11 minutes). The mean ART was 150 minutes. A positive correlation was again seen between ART and arrival to CT Time $(p=0.02)$. For every 10 -minute delay in CT imaging, there was a 9-minute delay in ART $(95 \% \mathrm{CI}$ 1 - 16 minutes).

Conclusions It is known that early initiation of both thrombolysis and ECR are associated with positive patient outcomes. There is a need to reduce time taken to complete CT imaging in regional Australia, as it is clearly shown to be associated with lengthened time for treatment initiation and timely referral. Reduction in this arrival to CT time will likely improve patient outcomes.

\section{TIME EQUALS BRAIN - RETROSPECTIVE ANALYSIS OF THROMBOLYSIS IN REGIONAL AUSTRALIA TO DETERMINE FACTORS WHICH INFLUENCE DOOR TO NEEDLE TIME}

Udit Nindra*, Toni M Wonson, Karen Fuller. Neurology Department, Wollongong Hospital, Wollongong, NSW, Australia

\subsection{6/jnnp-2019-anzan.28}

Introduction Minimising delay in thrombolysis is a key outcome in acute stroke care.

Methods A 3 year retrospective cohort analysis of all acute stroke admissions in Wollongong Hospital, a major regional referral centre in New South Wales, was completed to determine the causes of in-hospital delays for 